\title{
Studies of the UHECR Mass Composition and Hadronic Interactions with the FD and SD of the Pierre Auger Observatory
}

\author{
J. M. Carceller ${ }^{1, a}$, for the Pierre Auger Collaboration ${ }^{2, b}$ \\ ${ }^{1}$ Departamento de Física Teórica y del Cosmos, University of Granada, Granada, Spain. \\ ${ }^{2}$ Full author list and acknowledgments at: http://www.auger.org/archive/authors_2018_09.html
}

\begin{abstract}
.
With data on the depth of maximum $X_{\max }$ collected during more than a decade of operation of the Pierre Auger Observatory, we report on the inferences on the mass composition of UHECRs in the energy range $E=10^{17.2}-10^{19.6} \mathrm{eV}$ and on the measurements of the proton-air cross section for energies up to $10^{18.5} \mathrm{eV}$. We also present the results on $X_{\max }$ obtained using the information on the particle arrival times recorded by the SD stations allowing us to extend the $X_{\max }$ measurements up to $10^{20} \mathrm{eV}$. The inferences on mass composition, in particular using the data of the $\mathrm{SD}$, are subject to systematic uncertainties due to uncertainties in the description of hadronic interactions at ultra-high energies. We discuss this problem with respect to the properties of the muonic component of extensive air-showers as derived from the SD data.
\end{abstract}

\section{Introduction}

The Pierre Auger Observatory is the largest detector of ultra-high energy cosmic rays (UHECR) built so far. It comprises an area of $3000 \mathrm{~km}^{2}$ and features two different types of detectors: The Fluorescence Detector (FD) and the Surface Detector (SD). With the FD, a direct measurement of the depth of maximum of shower profiles, $X_{\max }$, is performed using the ultraviolet light emitted by nitrogen as the shower develops in the atmosphere. While the FD operates only during moonless nights and has a duty cycle of around $15 \%$, the SD has an almost $100 \%$ duty cycle. The SD measures the time of arrival of particles at the ground level and the signal produced by these particles by means of the Cherenkov light that particles emit when they pass through the water inside the stations of the SD. With its hybrid design, several techniques can be exploited to acquire information about mass composition. However, the interpretation of the results depends on the results of the simulations, and these depend on the choice of model for the hadronic interactions of high energy. For this reason, the study of hadronic interactions is of utmost importance for the correct interpretation of the results obtained in studies of mass composition.

\footnotetext{
ae-mail: jmcarcell@ugr.es

be-mail: auger_spokespersons@fnal.gov
} 


\section{Mass composition}

Mass composition is one of the fundamental questions that remain unsolved about ultra-high energy cosmic rays. Information about the mass composition of cosmic rays of such high energies can allow to constrain models about acceleration at the sources and to help to understand the propagation mechanisms to which these cosmic rays are exposed.

One of the most important observables for mass composition is the slant depth of maximum $X_{\max }$. $X_{\max }$ is the depth where the number of particles (or the energy deposition) reaches its maximum and is measured with the Fluorescence Detector (FD) [1].

In Figure 1, it can be seen that the average value of $X_{\max }$ is different in simulations using proton and iron primaries. It scales linearly with the energy so that for a constant composition the evolution of $X_{\max }$ with the energy given by the elongation rate is:

$$
D_{10}=\frac{\mathrm{d} X_{\max }}{\mathrm{d} \lg (E / \mathrm{eV})}=60 \mathrm{~g} / \mathrm{cm}^{2} / \text { decade }
$$

However, from data it is found that $D_{10}=79 \pm 1 \mathrm{~g} / \mathrm{cm}^{2} /$ decade between $10^{17.2}$ and $10^{18.33} \mathrm{eV}$. This result is an indication of a change in the primary mass from heavier to lighter because the elongation rate is significantly larger than for a pure composition. On the other hand, $D_{10}=26 \pm 2 \mathrm{~g} / \mathrm{cm}^{2} / \mathrm{decade}$ from $10^{18.33} \mathrm{eV}$ onward, indicating a change in composition from lighter to heavier. The fluctuations of $X_{\max }$ also decrease above $10^{18.3} \mathrm{eV}$, indicating a change in composition towards a heavier primary [2]. These results can be directly compared to simulations because they are obtained once all the effects related to the detector, such as the resolution of the detector or the acceptance, are taken into account.

The study of mass composition using $X_{\max }$ can be extended beyond the average value and the fluctuations. For this, the distributions of $X_{\max }$ in data have been matched with those in simulations. Simulations of showers with different primaries (namely proton, helium, nitrogen and iron) have been made, and the fraction of each primary has been left free to find the distribution that best approximates the distribution of $X_{\max }$ in data. The result is that models can not find a combination of fractions that can reproduce the details of the observed $X_{\max }$ distributions [2, 3] because the fraction of $p$-values obtained for the fits that are small $(<0.1)$ is significantly above what is expected on statistical grounds.

Information for mass composition can also be extracted from the measurements of the SD. Using the risetime of the signal measured in each station, $\Delta$ is defined as an observable that measures how fast or slow the risetimes of an event are compared to an average benchmark. This benchmark describes the behaviour of the risetime as a function of the distance of each station to the core of the shower. This observable can be calibrated in hybrid events with $X_{\max }$, and thus a value of $X_{\max }$ can be assigned to all the events, extending the current measurements of $X_{\max }$ up to $10^{20} \mathrm{eV}$. In Figure 2 the values of the $X_{\max }$ obtained with this technique (called the Delta method) are compared to those measured by the FD. These values have been transformed to $\langle\ln A\rangle$, the average logarithm of the mass number. The results obtained are compatible with what the FD measures, and at the highest energies the change in composition from lighter to heavier seems to be stopping, but uncertainties are large [4].

\section{Hadronic interactions}

Useful information can be extracted from cosmic-ray showers for the study of hadronic interactions. In particular, it can be proved that the tail of the distribution of $X_{\max }$ is sensitive to the proton-air cross section. This result, in combination with the Glauber model, has been successfully employed 

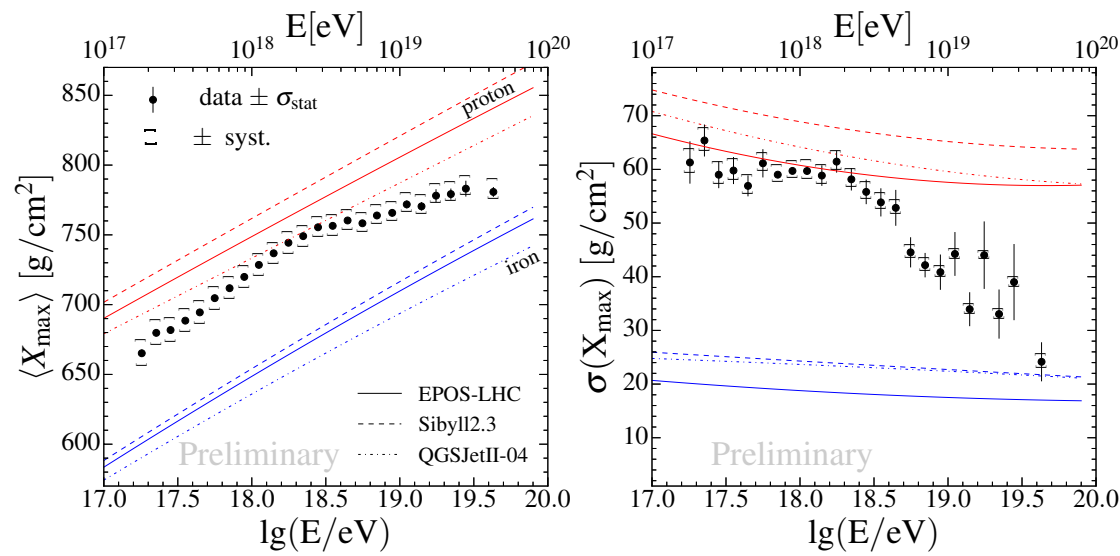

Figure 1. Evolution of the average value of $X_{\max }$ with the energy (left) and its standard deviation (right) for both data and simulations [2].

to obtain the proton-proton cross section at $\sqrt{s}=57 \mathrm{TeV}$, a center of mass energy that is higher than what current accelerators can achieve. The results for the inelastic and total cross section are [5]:

$$
\begin{aligned}
\sigma_{p p}^{\text {inel }} & =\left[92 \pm 7(\text { stat })_{-11}^{+9}(\text { sys }) \pm 7(\text { Glauber })\right] \mathrm{mb} \\
\sigma_{p p}^{\text {tot }} & =\left[133 \pm 13(\text { stat })_{-20}^{+17}(\text { sys }) \pm 16(\text { Glauber })\right] \mathrm{mb} .
\end{aligned}
$$

Some discrepancies have been found between the number of muons measured in data and simulations. Inclined events (zenith $\theta>60^{\circ}$ ) that have been measured at the same time by the FD and SD have been studied. The interest in these events is that most of the signal comes from muons due to the absorption of the electromagnetic component of the shower in the longer path of atmosphere that they have to traverse to reach the detector. It is found that it should be necessary to increase the number of muons between $30 \%$ and $80 \%$ (depending on the model used for high-energy hadronic interactions) to match with simulations what is measured in the data [6]. In the left panel of Figure 3, the average logarithmic muon content is compared to simulations, and it is found to be significantly above what is obtained from simulations.

Another way of showing this discrepancy is to study simulations that have the same longitudinal profile (dominated by the electromagnetic component) as in data. For this, simulations are made until a longitudinal profile that is very similar to that in the data is obtained. Then, the signal at the ground measured by the SD is studied as the sum of the contribution of an electromagnetic and a hadronic component:

$$
S_{\text {resc }}\left(R_{E}, R_{\mathrm{had}}\right)_{i, j} \equiv R_{E} S_{E M, i, j}+R_{\mathrm{had}} R_{E}^{\alpha} S_{\mathrm{had}, i, j},
$$

where $R_{E}$ is a factor that scales the electromagnetic component, $R_{E}^{\alpha}$ reflects the fact that the hadronic signal increases slower than linearly with energy, and $R_{\text {had }}$ is the scaling of the hadronic component. Then, $R_{E}$ and $R_{\text {had }}$ are left free in a fit to match the signal measured in data. In the right panel of Figure 3, it can be seen that while the energy rescaling $R_{E}$ is compatible with 1 , meaning that no scaling is needed, the scaling of the hadronic component is significantly above 1 for the hadronic models used and compositions shown [7]. 

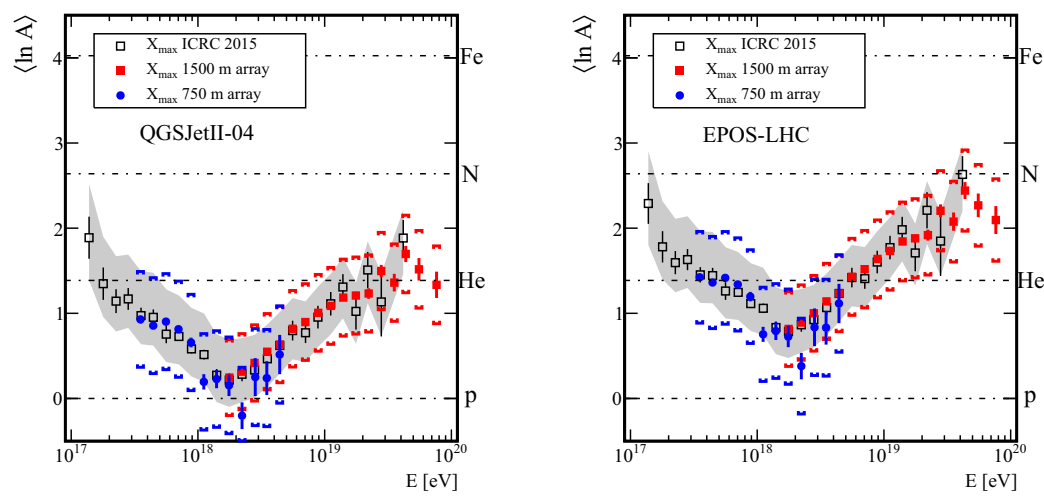

Figure 2. $\langle\ln A\rangle$ as a function of the energy. The results obtained with the Delta method (filled squares and filled circles) are compared to the values of $X_{\max }$ measured by the FD (open squares) [4].
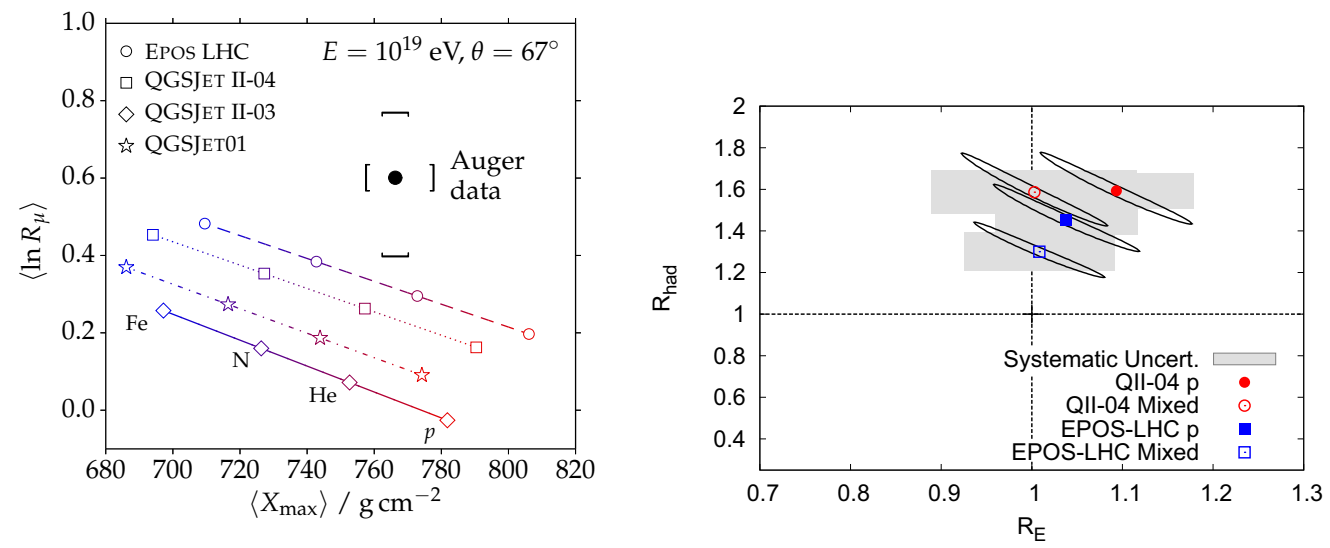

Figure 3. (Left) Average logarithmic muon content as a function of the average shower depth $\left\langle X_{\max }\right\rangle$. Model predictions are obtained from showers simulated for $\theta=67^{\circ}$ for proton and iron showers, and interpolated for helium and nitrogen showers [6]. (Right) Best-fit values of $R_{E}$ and $R_{\text {had }}$. The ellipses and grey boxes show the $1-\sigma$ statistical and systematic uncertainty [7].

\section{References}

[1] The Pierre Auger Collaboration, Phys. Rev. D 90, 122005 (2014), 1409.4809

[2] Jose Bellido for The Pierre Auger Collaboration, PoS (ICRC2017) 506 (2017)

[3] The Pierre Auger Collaboration, Phys. Rev. D 90, 122006 (2014), 1409. 5083

[4] The Pierre Auger Collaboration, Phys. Rev. D 96, 122003 (2017), 1710.07249

[5] The Pierre Auger Collaboration, Physical Review Letters 109, 062002 (2012), 1208 . 1520

[6] The Pierre Auger Collaboration, Phys. Rev. D 91, 032003 (2015), 1408. 1421

[7] The Pierre Auger Collaboration, Physical Review Letters 117, 192001 (2016), 1610.08509 